\title{
Learning epidemic threshold in complex networks by Convolutional Neural Network
}

\author{
Qi Ni, ${ }^{1}$ Jie Kang, ${ }^{1}$ Ming Tang, ${ }^{2,3}$, * Ying Liu, ${ }^{4,5}$, and Yong Zou ${ }^{6}$ \\ ${ }^{1}$ School of Communication and Electronic Engineering, \\ East China Normal University, Shanghai 200241, China \\ ${ }^{2}$ School of Mathematical Sciences, Shanghai Key Laboratory of PMMP, \\ East China Normal University, Shanghai 200241, China \\ ${ }^{3}$ Shanghai Key Laboratory of Multidimensional Information Processing, \\ East China Normal University, Shanghai 200241, China \\ ${ }^{4}$ School of Computer Science, Southwest Petroleum University, Chengdu 610500, China \\ ${ }^{5}$ Big Data Research center, University of Electronic Science and Technology of China, Chengdu 610054, China \\ ${ }^{6}$ School of Physics and Electronic Science, East China Normal University, Shanghai 200241, China
}

(Dated: November 19, 2019)

\begin{abstract}
Deep learning has taken part in the competition since not long ago to learn and identify phase transitions in physical systems such as many body quantum systems, whose underlying lattice structures are generally regular as they're in euclidean space. Real networks have complex structural features which play a significant role in dynamics in them, and thus the structural and dynamical information of complex networks can not be directly learned by existing neural network models. Here we propose a novel and effective framework to learn the epidemic threshold in complex networks by combining the structural and dynamical information into the learning procedure. Considering the strong performance of learning in Euclidean space, Convolutional Neural Network (CNN) is used and, with the help of confusion scheme, we can identify precisely the outbreak threshold of epidemic dynamics. To represent the high dimensional network data set in Euclidean space for CNN, we reduce the dimensionality of a network by using graph representation learning algorithms and discretize the embedded space to convert it into an image-like structure. We then creatively merge the nodal dynamical states with the structural embedding by multi-channel images. In this manner, the proposed model can draw the conclusion from both structural and dynamical information. A large number of simulations show a great performance in both synthetic and empirical network data set. Our end-to-end machine learning framework is robust and universally applicable to complex networks with arbitrary size and topology.
\end{abstract}

The combination of machine learning and complex networks isn't a new topic since a variety of graph representation learning techniques and deep learning based graph neural networks have found their applications in the fields of network embedding, node classification, link prediction and network reconstruction, etc. However, these research frontiers focus on learning the topology instead of dynamics of the networks. Deep learning algorithms have been applied in learning and identifying the dynamics in Ising model these years. Inspired by previous researches, we propose in this work a robust and efficient machine learning framework to identify the epidemical threshold of SIS dynamics in complex networks. Convolutional Neural Network (CNN) plays a key role of learner in the framework and the input networks are converted into $2 \mathrm{D}$ imagelike structures, on which CNN performs well. We then creatively add dynamical information into the images and thus our model can learn the networks by gathering the structural and dynamical information together. A large number of experiments on both synthetic and empirical net-

\footnotetext{
* tangminghan007@gmail.com

† shinningliu@163.com
}

works prove that the proposed framework is accurate and universally applicable. The proposition of our framework opened a new perspective to learn and identify critical point of dynamics in complex networks, using machine learning techniques. Our work combines both topological and dynamical information of a network, exploring a new way to solve the threshold-related issues.

\section{INTRODUCTION}

Deep learning is an interdisciplinary frontier as researches on computer vision, natural language processing, recommendation system, etc., thrive [1. This benefits much from the increasing availability of computational resources and easiness to access massive data set. Recently, this topic has attracted much attention from physicists because of the strong performance of detecting, predicting and uncovering various phases of matter in quantum many-body systems $2-13]$. Neural network based models play an irreplacable role in this field because they're able to not only learn generate phases or states of matter that are previously known $4,5,7,12$ or uncovering phase transitions [2, 3, 6, but predict out-ofequilibrium phases of matter that have not been known yet [13. Both Feed-Forward Neural Network (FFNN) and Convolutional Neural Network (CNN) have been ex- 
ploited to learn and discover the hidden pattern of phase transition in Ising-type lattice structures [7. Although FFNN can give a good prediction sometimes, it is usually outperformed by CNN because the latter is able to store and learn the topological information of the system. It was demonstrated that the threshold or the critical point phase transition in the Ising model can be predicted through deep learning [14]. All these accomplishments benefit greatly from the Euclidean topology of the underlying Ising spin lattice.

In recent years, researches have achieved unprecedented results on a large variety of natural, social, and engineering systems [15. A complex network, by definition, has a complex topology and, as a natural phenomenon associated with network dynamics, the emergence of distinct phases and phase transitions are ubiquitous [16]. Though there have been a massive number of works incorporating machine learning into the field of complex networks [17 21], these studies were confined to learning the structural information of the network. Network representation learning is a good example in which a network is dimensionally reduced with most of its structure information intact. There are applications in problems such as link prediction [22], clustering [23], and node classification [24. Based on random walk and graph search algorithm, the algorithm named "node2vec" can embed the network topology into a lower dimensional space by integrating macroscopic and microscopic structural information of the network [18. The algorithm "Deepwalk" finds a way to unite skipgram, a natural language processing technique, with random walk sequences on graphs [17, providing a concise solution to graph embedding. Furthermore, it was proved that the performances of deep learning models such as Graph Convolutional Networks (GCN) [19] and structural deep network embedding 20 can be equivalent to those of traditional, random-walk based methods.

The key aspect that distinguishes our work from the previous works is that we exploit machine learning to deal with dynamics responsible for phase transitions in complex networks. To be concrete, we exploit deep learning, together with confusion scheme [6] to identify phase transitions of epidemic spreading in complex networks 25 32. There is a typical second-order phase transition in this dynamical process. It is an active research topic to precisely learn and identify the critical point of the phase transition. A widely applied method is the so-called "degree-based mean field" approach [16, 25], which gives the theoretical threshold as $\langle k\rangle /\left\langle k^{2}\right\rangle$, where $\langle k\rangle$ and $\left\langle k^{2}\right\rangle$ are the first and second moments of the degree distribution, respectively. However, the theoretical prediction is not always accurate because of the dynamical correlations among nodes. Thus for real world networks, there must be a discrepancy between the calculated and simulation results. It is commonly agreed that the results obtained by simulation are accurate enough by using Monte-Carlo simulations through measures such as network susceptibility [33, variability [34] or the av- erage lifetime [35]. The basic idea and working principle of our machine learning based approach differ fundamentally from the existing methods.

We've made a significant step forward to make the deep learning model learn and identify the critical point of phase transition [36. However, FFNN fails to take the network topology into consideration and the structural information is omitted at the very beginning in the input layer. To overcome the disadvantage, a couple of sampling methods based on nodal importance are proposed to manually "restore" the missing topological information. Inspired by our previous work, we use CNN to learn both topological and dynamical information in networks and make the prediction all by itself without any additional operation and feature engineering. Also, our CNN based model outperforms our previous model and no sampling method is needed to get topological information.

The rest of the work is organized as follows: in section III we focus on the susceptible-infected-susceptible (SIS) epidemic spreading model and the structure and workflow of the proposed machine learning framework. In section III. we introduce hyperbolic embedding in complex networks and explain in detail why it is needed in our work, together with the experimental results in synthetic networks. In section [V] we show the results and performances of the proposed model in real-world networks. Finally in section $\mathrm{V}$, we take a review of our contribution in this work and provide a couple of open questions to inspire further researches.

\section{PROPOSED MODEL AND METHOD}

In this section we introduce the SIS spreading model, as well as the proposed CNN based machine learning framework and its training data set.

\section{A. Epidemic spreading and measure of susceptibility}

In this work, the classical SIS epidemic process [15] is used. At each time step, an infected node transmits the disease to its susceptible neighbors with infection rate $\beta$, and the infected node returns to susceptible state with recovery rate $\mu$. As we know, there are two phases in the spreading process: active and absorbing state. In active state there are both two types of nodes in the network while in absorbing state, there is no infected node. The effective infection rate is defined as the ratio of the infection rate to the recovery rate, which is $\lambda=\beta / \mu$, and the critical point of $\lambda$ can be denoted as $\lambda_{c}$. The system approaches the absorbing state after a period of time if $\lambda \leq \lambda_{c}$. For $\lambda>\lambda_{c}$, the system will reach an endemic state where the density of the infected nodes remains stable. Under this circumstance, the system is in the active phase. 
The numerical threshold of the SIS process can be characterized by susceptibility [33] defined as

$$
\chi=N \frac{\left\langle\rho^{2}\right\rangle-\langle\rho\rangle^{2}}{\langle\rho\rangle},
$$

where $\rho$ is the density of the infected nodes (i.e., the order parameter), $N$ is the network size, $\langle\rho\rangle$ and $\langle\rho\rangle^{2}$ are the first and second moments of $\rho$, respectively. The susceptibility measure indicates the variation in outbreak sizes over many runs with the same parameter $\lambda$. The order parameter associated with the second-order phase transition ( $\rho$ in this case) shows a power-law distribution near the critical point. Hence the variation of the order parameter reaches the peak at this point and so does the susceptibility curve. As a function of the effective infection rate $\lambda$, the susceptibility measure reaches its maximum value at $\lambda_{c}^{\chi}$, which is the threshold of the epidemic process or the critical point of phase transition. In machine learning, especially for supervised learning, what we need is a measure to accurately reflect the ground truth of the transition point and the corresponding label during training can be obtained. The susceptibility measure plays an important role under this circumstance.

\section{B. Proposed machine learning framework: overview}

We propose a machine learning framework to learn the SIS dynamics and identify the critical point of the phase transition. We turn a network into an image-like regular structure with nodal dynamical information added by multiple channels. The CNN can take these image-like data as input and finally output a probability indicating how possible the input data is in one specific phase. These outputs are compared with man-made fake labels and the critical point can thus be inferred by confusion scheme [6], a powerful tool combining ideas from both supervised and unsupervised learning. For simplicity, the workflow can be decomposed into four steps as illustrated in Fig. 1:

(1) Embed network into two dimensional space.

(2) Discretize network embedding and add dynamical information.

(3) Learn by $2 \mathrm{D}-\mathrm{CNN}$.

(4) Identify the critical point using confusion scheme. In the following text we introduce in detail the structure and workflow of our model and explain why it is powerful in the given task.

\section{Details of proposed method}

\section{How to represent network data as image-like structures?}

We aim to convert the high dimensional network data into 2D lattice, a two dimensional Euclidean space discretized rectangles. A 2D lattice meets the condition of spatial dependence [37, 38, which indicates everything is related to everything else, but near things are more related than distant things. In a context of images, the closer the pixels are, the more related they become. Consider a network denoted as $G(V, E)$ where $V$ and $E$ represent the node and edge sets, respectively. Classically, this highdimensional structure can be represented by its adjacency matrix $A$ or Laplacian matrix $L$. Adjacency matrix is a square $(N, N)$ matrix where $N$ is the size of $V$. It is symmetric in unweighted graphs and the $(i, j)^{t h}$ entry $A_{i j}$ is the weight between two nodes $i$ and $j$ if there is an edge $e_{i j}$ between them, or 0 otherwise. The Laplacian matrix can be obtained by $L=D-A$, where $D$ is the diagonal degree matrix. The eigenvalue and eigenvector of $L$ contains hidden information of the network. $L$ is largely used in network representation learning algorithms based on matrix decomposition. To convert this kind of data into an image, we do the following steps:

Graph embedding. First, we use a graph representation learning algorithm to embed the network data into a low dimensional underlying space. The feature extracted from the network is the structural similarity between nodes, which is indicated by the hyper distance of any two node representations. For instance, neighbors tend to be close in the latent space compared to non-neighbors. We get the representation by Deepwalk [39, which is a graph embedding algorithm based on random-walk. As many state-of-the-art network representation algorithms (e.g., Deepwalk) are neural, they are stochastic. A given dimension will not be associated with the same latent space across networks or across several runs on the same network. Therefore, PCA [40] is used to ensure these embeddings are comparable. The PCA also serves an information maximization (compression) 37 purpose as it greatly reduces the size of tensors fed to the CNN. In our work, PCA converts the embedded data to 2-dimensional images which will be the input of CNN later. Figure 2 (a) shows the image which is obtained by embedding a random regular (RR) network (with $N=1000$ ) into twodimensional space.

Stacking node representations in 2D histograms. Since the $2 \mathrm{D}$ representation space is continuous and not able to be fed to CNN, we take a step forward to transform the space into finite sized lattice, making it learnable for CNN. We first divide the space into rectangle sub-regions and then color each sub-region by counting the number of nodal representations falling into it. Accordingly, a grayscale image is generated. As is represented in Fig. 2 (b), the BA network is converted into a grayscale image. The brighter a region is, the more nodes there are. After discretization, the graph image turns to a stack of histograms representing the number of nodes in each subarea. It is worth noticing that the final dimensionality does not depend on the size of the network (e.g., number of nodes or edges). Networks with arbitrary size will be converted into images of the same size.

Adding nodal dynamical information. One of the key contributions of our work is that we merge the nodal 


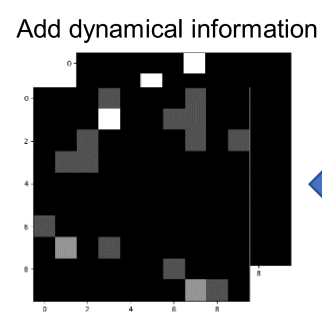

(f)
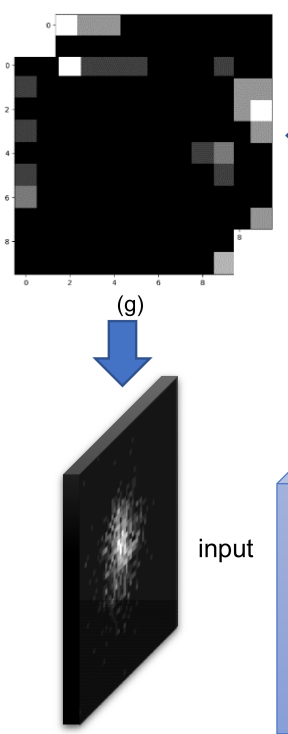

Discretize network embedding

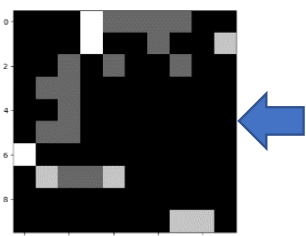

(d)

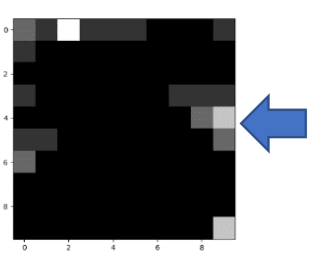

(e)

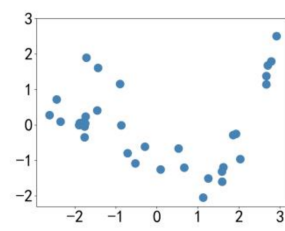

(b)

Poincaré embedding

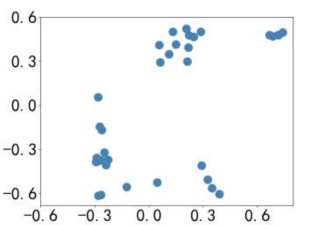

(c)

FFNN CONV3-32

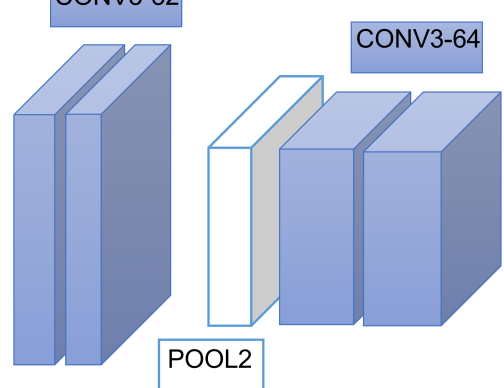

(h)
Input network
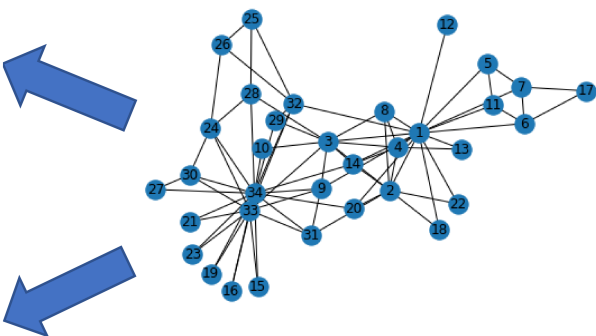

(a)

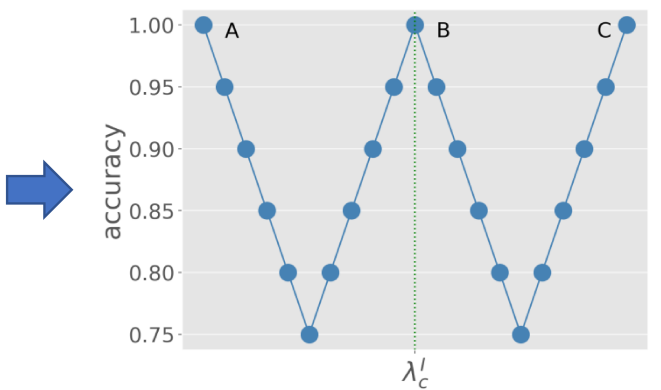

(I)

FIG. 1. Workflow of the proposed machine learning model. (a) Input network, which is represented by adjacency matrix sized $N * N$, with the $N$ denoting the number of nodes. (b, c) Network representation learning. Network dimensional reduction algorithms and PCA are used to reduce the dimensionality of the input signal to 2. (d, e) Discretize the continuous embedded data into histograms, as this image-like structure makes the following learning procedure possible. The color of each rectangle sub-region represents the number of nodes falling into it. (f, g) Adding dynamical information. We combine the structural and dynamical information by split the single-channel images into two channels, representing the nodes in the state of $S$ and $I$, respectively. (h) The architecture of CNN we use. It consists of two convolution layers with 32 and 64 filters, respectively. Following every convolution layer is a $(2,2)$ max pooling layer. The second convolution-pooling block sends its output to a 128-neuron dense layer to calculate the probability that the input data is supposed to be in a specific phase. All of these above are constructed with tensorflow. (I) Schematic illustration of confusion scheme to detect phase transition and identify the epidemical threshold. For a range of tentatively assigned threshold values, the curve of classification accuracy of supervised learning versus the threshold value will exhibit a $W$-shape, if there is a phase transition associated with epidemic dynamics on the network. The location of the middle peak gives an accurate prediction of the true threshold value. If the network dynamics do not exhibit a phase transition, the curve would exhibit a $U$ shape (see text for a detailed reasoning).

dynamical information into the $2 \mathrm{D}$ images. As there are two states in the dynamics of SIS, we split our image into multiple channels, with each channel represents one kind of nodal dynamical state information. For SIS, we split it into two channels and there are only histograms of nodes in state $I(S)$ in every single channel. Fig. 1 (f) and (g) show the two-channel representations of the original network with nodal dynamical states. In this manner, CNN will be able to learn the structural and dynamical knowledge from the input data set, and make a better decision than merely learn from part of them.

How does CNN learn from images and what is

\section{the data to be learned?}

Deep learning on networks is a hot topic these years as graph neural networks show the great performance on nodal semi-classification, link prediction, network reconstruction, etc. 41 44. GCN is a simple but powerful model 44 which is able to learn from network structure and is widely used in a variety of network-related fields. However, the GCN is unable to learn the dynamical information. What's worse, the adjacency matrix is needed to train the GCN and hence it is difficult to be applied in large-scale networks. We take a very different way to learn the network's structural and dynamical 

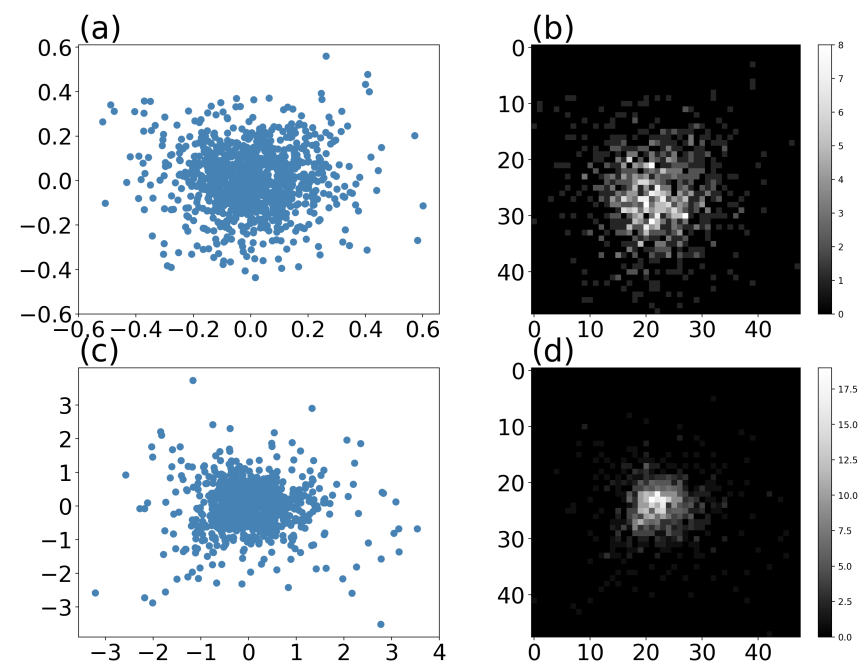

FIG. 2. Embedding network into two-dimensional space with deepwalk. (a, c) Embed an RR network with $N=1000$ and $\langle k\rangle=10$ (a) and BA network with $N=1000$ and $m=m_{0}=3$ (c). Deepwalk is used to generate the $d$-dimensional $(d \ll N)$ representation for each node first. To reduce the stochasticity of neural based algorithms, we use the PCA to extract two principal components from the embedding space and greatly decrease the input size to CNN. The horizontal and vertical axises represent the first and second principal component, respectively. (b, d) Discretize the embedding spaces and convert them into grayscale images. To transform our embedded data into image-like structure, we split the space into rectangle sub-region and color each region according to the number of nodal representations in it (denoted by color bar). In this work, we create images with the resolution of $48 * 48$, which means there're 48 sub-regions in a row (column).

information at a time by converting the input network into multi-channel, image-like 2D structures with CNN. A CNN consists of three kinds of layers: the convolution layer focuses on extracting information from its input by a continuous sliding convolutional kernel; the pooling layer tries to reduce the size of data which will be feeded into next layer and to reduce the risk of over fitting; and the fully-connected layer classifies the data into multiple "groups". In the context of our work, there're two "groups" representing two different phases of the system.

In this work, the CNN contains four convolution layers, two max-pooling layers, one fully-connected layer and one output layer. As is shown in fig 1 (h), the sizes of the convolutional filter (kernel) and pooling operator are $3 * 3$ and $2 * 2$, respectively. For the first convolution-pooling block, 32 filters are employed in the convolution layer, following with a $(2,2)$ max pooling layer which halves the input signal. The number of filters in the subsequent convolution layer is increased to 64 to compensate the loss of resolution. After the feature extraction process, the data enters a 128-neuron dense (fully connected) layer and the output layer will give the probability that the input data is supposed to be in a specific phase. Since our model is not sensitive to hyper-parameters, we de- cide these hyper-parameters, together with some others, by a coarse grained grid search. We don't do a further "fine tuning" as the learning results have already been "good". Dropout [45] and $L 2$ regularization are used to prevent over fitting.

We use synchronous updating Monte Carlo simulation to obtain the training and test data set for CNN. For the underlying neural network to learn the system dynamics, proper inputs are needed. In an absorbing phase, the nodal states are all identical (all 0 in data set), rendering them improper for training. Near the phase transition point, i.e., when the value of $\lambda$ is in the vicinity of $\lambda_{c}$ $\left(\left|\lambda-\lambda_{c}\right| \gtrsim 0\right)$, there is a high probability for the system to be trapped in the absorbing state. To overcome this, we use the quasi-stationary method [46] to prevent the system from entering the absorbing state. Specifically, whenever the system tends to enter the absorbing state, we change its state to that of the previous time step. This simple procedure allows us to obtain the legitimate training and test sets for learning.

To make it more specific, consider the data set is basically ordered along a tuning parameter $\lambda$, which is also the control parameter of the phase transition. The data with a certain value of $\lambda$, will be described by $\mathbf{s}(\lambda)$ and it consists of a set of grayscale images, each of which represents the distribution of nodal 2D representations in the embedding space. Nodal dynamical states are added to images by multi-channel. There are only susceptible (infected) nodes in each channel. The brightness of each rectangle sub-region indicates the amount of nodes in it. The neural network is thought to be a high-level mapping function that takes data $\mathbf{s}(\lambda)$ to infer the probability distribution $\mathcal{F}(\mathbf{s}(\lambda))=p_{0}$, where $p_{0}\left(1-p_{0}\right)$ represents the inferred probability that $\mathbf{s}(\lambda)$ is in the phase of absorbing (active) state. This can be considered directly with the probability $L(\lambda)=H\left(\lambda-\lambda_{c}\right)$, where $\lambda_{c}$ is the ground truth (fake truth) that is preset in advance and $H$ represents the unit step function $H(x)=\frac{d}{d x} \max \{x, 0\}, x \neq 0$. In the training procedure, a large data set of different $\mathbf{s}(\lambda)$ and corresponding labels $L(\lambda)$ are fed to the neural network and this network makes $\mathcal{F}(\mathbf{s}(\lambda)) \rightarrow L(\lambda)$ by optimizing its parameter set $\mathbf{P}_{\mathcal{F}}$ to minimize a cost function $C(\mathcal{F}(\mathbf{s}(\lambda)), L(\lambda))$. The cost function evaluates the gap between the network's judgment and the real answer quantitatively. It can be minimized through a lot of optimization method such as stochastic gradient descent or $A D A M$ 47. In this work, input data are a set of $(48,48,2)$ images (48 for resolution and 2 for number of channel) and the corresponding labels are a vector of 0 and 1 , representing absorbing and active phase, respectively.

\section{How are the critical point inferred by confusion scheme?}

As is demonstrated in the previous sub-section, it is essential to have a correct set of labels to make the CNN draw an unbiased conclusion. However, in some cases the label, which is supposed to be a kind of prior knowledge, cannot be easily obtained or guaranteed flawless. These 

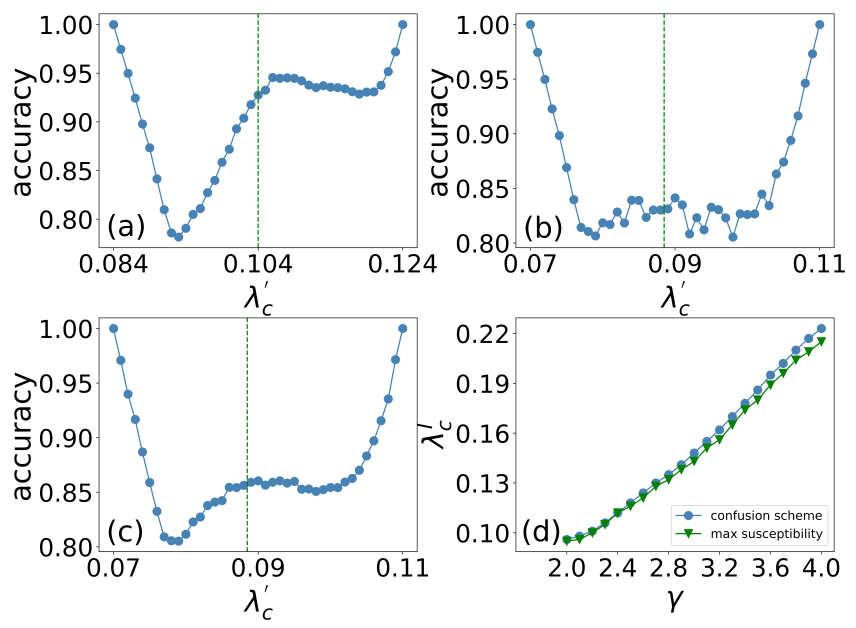

FIG. 3. Learning phase transition on homogeneous and heterogeneous networks with deepwalk. (a) For a random regular network of size $N=1000$ and average degree $\langle k\rangle=10$, over model generates an overall $W$-shape curve of the classification accuracy versus the assumed threshold value. The peak of susceptibility is at 0.104 , as indicated by the green vertical line (the same legend holds below). (b) For a $B A$ network of size $N=1000$ and $m=m_{0}=3$, the neural network fails to yield a $W$-shape accuracy curve: the accuracy has a $U$-shape. (c) For the same $B A$ network as (b), the accuracy curve obtained by incorporating Poincaré embedding into the training process. In this case, the accuracy curve exhibits a $W$-shape, rendering detectable the phase transition. The transition point can also be identified accurately. (d) For scale-free networks generated by the uncorrelated configuration model (UCM) of size $N=1000$, max degree $k_{\max }=\sqrt{N} \approx 32$ and the power law exponent $\gamma$ in the range $[2.0,4.0]$, the epidemical thresholds obtained by susceptibility measure and our model are shown. Blue circles and green triangles represent the results given by susceptibility and our model, respectively.

dirty labels will significantly decrease the performance of the model [36. We use the confusion scheme that can make precise prediction without any prior knowledge about the labels [6]. The only difference between confusion scheme and original supervised learning is that the labels of confusion scheme method are man-made guesses instead of ground truths. As our data are indexed by order parameter $\lambda$, we can avoid using any prior knowledge by making a "good guess" of the true transition point. The number of candidates in total is $\mathcal{N}+1$ where $\mathcal{N}$ is the number of different values of $\lambda$ existing in our data set. It's completely affordable to perform such a "brute force" guess and the result can be inferred by our neural network.

Figure 1 (I) shows how the confusion scheme work in detail. Suppose we have a set of unlabeled network configuration data ranging from $\lambda_{\min }$ to $\lambda_{\max }$. Let $\lambda_{c}$ be the unknown true threshold value, where $\lambda_{\min } \leq \lambda_{c} \leq \lambda_{\max }$. We assign tentative labels to the data set by assuming that the threshold is $\lambda_{c}^{\prime}$, where $\lambda_{\min } \leq \lambda_{c}^{\prime} \leq \lambda_{\max }$. We assign label zero to all configurations for $\lambda \leq \lambda_{c}^{\prime}$ and label one to those with $\lambda>\lambda_{c}^{\prime}$. We then choose a number of closely located values of $\lambda_{c}^{\prime}$. For each value of $\lambda_{c}^{\prime}$, we conduct the training and obtain the classification accuracy. Ideally, we expect the accuracy to exhibit a $W$-shape curve versus $\lambda_{c}^{\prime}$. For $\lambda_{c}{ }^{\prime}=\lambda_{\text {min }}$, every row of the training set is labeled as one, so the neural network regards the data of every pattern as in the activation phase, giving rise to $100 \%$ accuracy of prediction. Similar result is expected for $\lambda_{c}^{\prime}=\lambda_{\max }$. For $\lambda_{c}{ }^{\prime}=\lambda_{c}$, the method reduces to supervised learning because the tentative or "fake" labels happen to be correct under the circumstance. The neural network can yield a high classification accuracy in this case [36]. For $\lambda_{\min }<\lambda_{c}{ }^{\prime}<\lambda_{c}$ or $\lambda_{c}<\lambda_{c}{ }^{\prime}<\lambda_{\max }$, the neural network will be "confused" for some data whose labels are exactly opposite to the true values, thereby leading to a decrease in the accuracy. Overall, a $W$-shape of the dependence of the accuracy on the value of $\lambda_{c}^{\prime}$ occurs, where the location of the peak in the middle corresponds to the identified threshold. If there is no phase transition in the threshold range $\left[\lambda_{\min }, \lambda_{\max }\right]$, the accuracy versus $\lambda_{c}^{\prime}$ would exhibit a $U$ shape. The emergence of a $W$-shape curve is thus an indication that there is phase transition in the system and the correct transition point (or threshold) can be identified accordingly without requiring any prior knowledge about the labels.

\section{THE NECESSITY OF HYPERBOLIC EMBEDDING}

To gain insight, we test our machine learning framework in both homogeneous and heterogeneous complex networks in Fig. 3 . Figure 3 (a) shows that our method can successfully identify the threshold in random regular networks with mean degree $\langle k\rangle=10$. Numerical simulation of the SIS dynamics reveals a second-order phase transition at $\lambda \approx 0.1040$ while the confusion scheme yields an overall $W$-shape behavior and the position of the middle peak occurs at $\lambda_{c}^{\prime} \approx 0.1050$, which is indistinguishable from the actual threshold value. In general, the proposed framework is quite effective for detecting phase transition with an accurate identification of the epidemical threshold for homogeneous networks.

For heterogeneous networks such as scale-free and $B A$ networks, this learning model is not always effective. As demonstrated in Fig. 3 (b), the accuracy curve does not exhibit an apparent $W$ shape in the chosen threshold range, whereas an actual phase transition occurs at 0.0893. A possible reason is that, in a typical heterogeneous network, the majority of nodes turn out to gather in a small area of the central part of the embedding space, as illustrated in Figs. 2 (c) and (d). This pattern may lead to the decrease of information and thus the learning performance deteriorates. It is known that the random walk based algorithm like deepwalk evaluates the similarity between two nodes by the probability that one node exists in the random walk sequence of the other node. For 

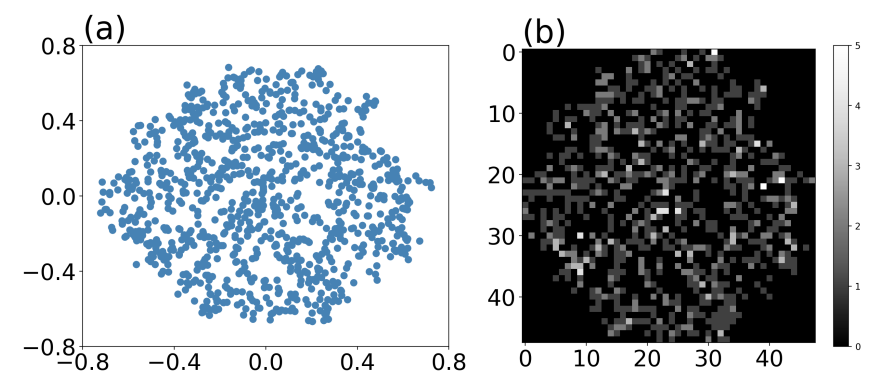

FIG. 4. Embedding network into two-dimensional space with Poincaré embedding. (a) Embed BA network with $N=1000$ and $m=m_{0}=3$ into a two dimensional hyperbolic disk. Each blue node in the figure is a two dimensional vector representing a vertex in the original network in the embedded space. (b) Discretize the embedding spaces and convert them into grayscale images. To transform our embedded data into image-like structure, we split the space into rectangle subregion and color each region according to the number of nodal representations in it (denoted by color bar). In this work, we create images with the resolution of $48 \times 48$, which means there're 48 sub-regions in a row (column).

scale-free networks like $B A$ model, there are often nodes of small degree in the neighborhood of a hub. In this way, it is possible that the deepwalk considers these two types of nodes similar during multiple round of training. It leads to the existence of the previously mentioned pattern. One possible solution is to increase the resolution of the image. We've tested the scenario where images are sized $(128,128,2)$ instead of $(48,48,2)$ and the $W$ shape can be obtained. However, because the time complexity against the resolution of images is quadratic, the computational cost increases sharply as the image resolution grows, implying it is impossible to be used in large scale networks.

Hence, a better embedding algorithm is needed to make the image look less "crowded". Next we pay attention to hyperbolic embedding. In mathematics, a hyperbolic space is a homogeneous space that has a constant negative curvature [48]. Hyperbolic space is suitable to model hierarchical data and this pattern is existing in complex networks widely, especially in realworld networks. Many previous works represent networks in hyperbolic disk [49, 50, where the relationship between Euclidean and hyperbolic distance $r_{e}$ and $r_{h}$ is $r_{e}=\tanh _{h} / 2$. It means that for a fixed Euclidean distance, hyperbolic distance in the peripheral area is much "longer" than it is in the central area of the disk. Here we exploit Poincaré embedding which embeds the high dimensional networks into a low dimensional Poincaré ball model [51. Figure 4 represents the image-like structure obtained by Poincaré embedding. As is shown, the nodes in the embedded space distributed equally in the embedding space instead of gathering in the central area, reducing the information loss compared to those with deepwalk algorithm [Figs. 2 (c) and (d)].

Figure 5 describes another reason why the Poincaré embedding outperforms the random walk based method. It is known that in the SIS spreading process, the hub nodes are the main spreaders. Nodes with large degree are considered more important than those with small degree [52], and the latter have little chance to be infected multiple times which accordingly provides less dynamical information than hubs. In an ideal scenario, we want to maximize the information given by nodes with large degree thus making the learning easier for CNN. Figure 5 (a) and (b) represent the relationship between nodal degree and average Euclidean distance of nodes to the center of the 2D embedding space. For Deepwalk, both the nodes with small and large degrees tend to locate in the central area of the space, which deteriorates the influence of hubs. In the discretized images with dynamical states, the central area and the periphery will both be dim. However, for the Poincaré embedding, hubs and leaves are distributed in the center and periphery, respectively. This pattern strengthens the influence of large-degree nodes: first, nodes do not gather in a very small area which reduces the information loss; second, hubs are in the central area and a bright spot is formed. In this way CNN will learn more dynamical information and accordingly get a better performance.

As represented in Figs. 3 (c) and (d), we test Poincaré embedding in BA and scale-free networks. In Fig. 3 (c), for the same BA network as in Fig 3 (b), the confusion scheme can successfully detect the phase transition and identify the critical point. In Fig. 3 (d), for scale-free networks generated by uncorrelated configuration model (UCM) with size $N=1000$ and the power law exponent $\gamma$ in the range $\gamma \in[2.0,4.0]$, with the help of Poincaré embedding, our machine learning framework does a great job in identifying the epidemic threshold accurately.

\section{APPLICATION TO REAL WORLD NETWORKS}

We test the performance of our model in real-world networks. Figure 6 illustrates the results for two realworld datasets. Although Deepwalk can get the final results sometimes, we use Poincaré embedding as it is well suited for processing hierarchical topology, which is a common feature in real-world complex networks. Results in more real-world networks are shown in Fig. 7. It is proved that our model gives a very accurate identified epidemical thresholds in almost every network. Details of the real-world data set we use and the corresponding results are listed in the appendix:

\section{CONCLUSION AND DISCUSSION}

In this paper, we propose a machine learning framework to identify the threshold of epidemic dynamics in complex networks and find that this method performs pretty well on a variety of synthetic and empirical net- 

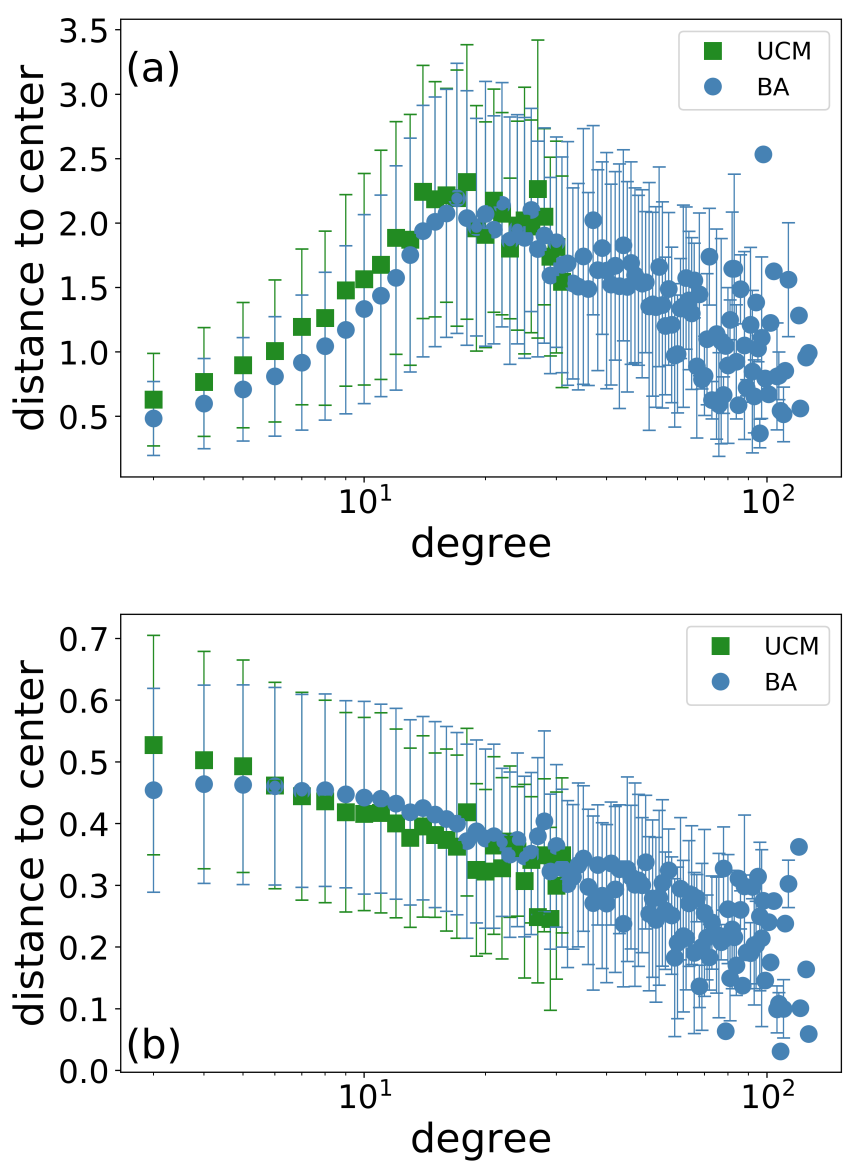

FIG. 5. Nodal distribution in the 2D embedding space of (a) Deepwalk and (b) Poincaré embedding for $B A$ networks of size $N=1000$ and $m=m_{0}=3$ (blue circles) and scale-free networks with $\gamma=2.5$ and $N=1000$ generated by UCM (green squares). Axises $x$ and $y$ represent the degree of nodes and the average Euclidean distance from nodes with a specific degree to the center of the space.

work topologies. The framework is end-to-end, computationally efficient, robust and universally applicable. We first convert the high dimensional networks into 2D images, in which the graph representation learning algorithms play an important role. While random walk based Deepwalk is simple and fast in computation, it fails to generate good pattern for CNN in some heterogeneous networks. Hence, the hyperbolic embedding method is introduced and applied on a large amount of network topologies. With its powerful ability in maximizing the information of hubs and representing networks with a hierarchical structure, Poincaré embedding is exploited in the majority of cases. We creatively add nodal dynamical states into the image with multiple channels: each channel represents the sub-network consisting of nodes in the state of $S(I)$. In this way, the following CNN is capable of learning dynamical and structural information of the network at a time. The CNN outputs a classification probability for input images with each $\lambda$ and finally the
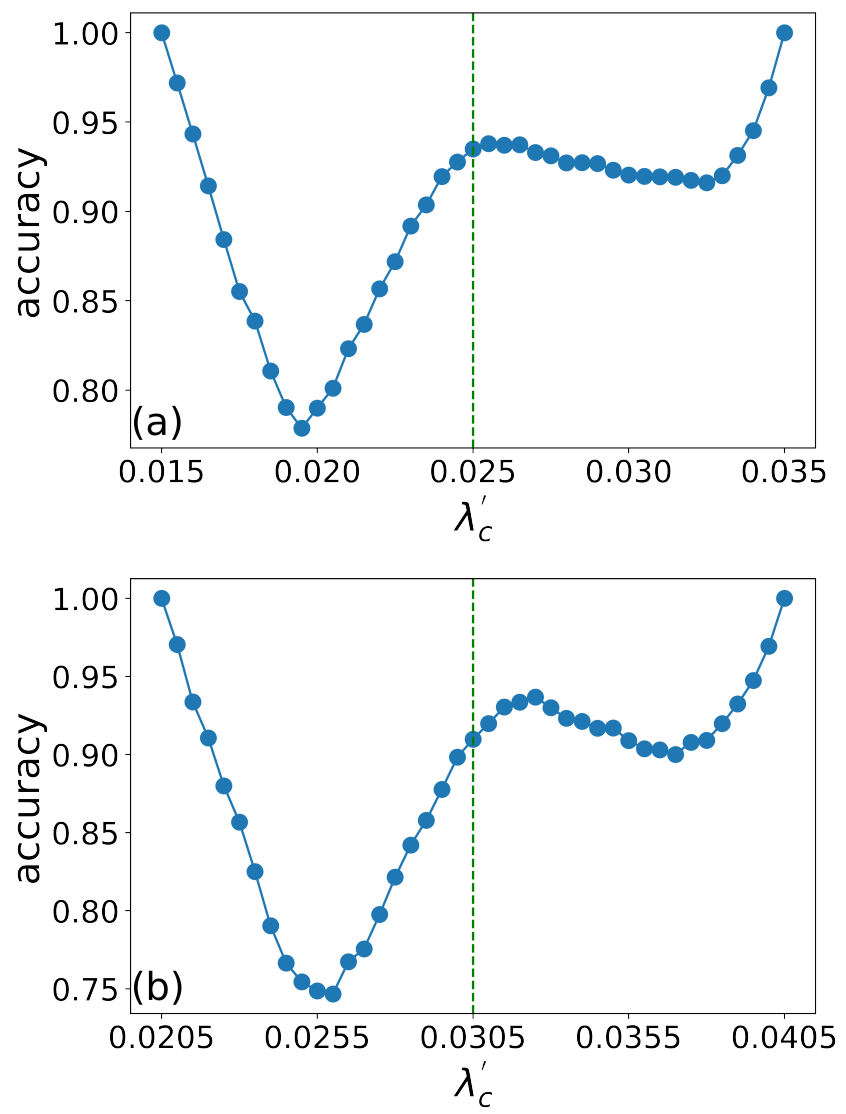

FIG. 6. Performance in two real-world networks. (a) Petster Friendship Hamster data set. (b) Pretty Good Privacy data set. For the details of these empirical networks and the corresponding results, please see the appendix.

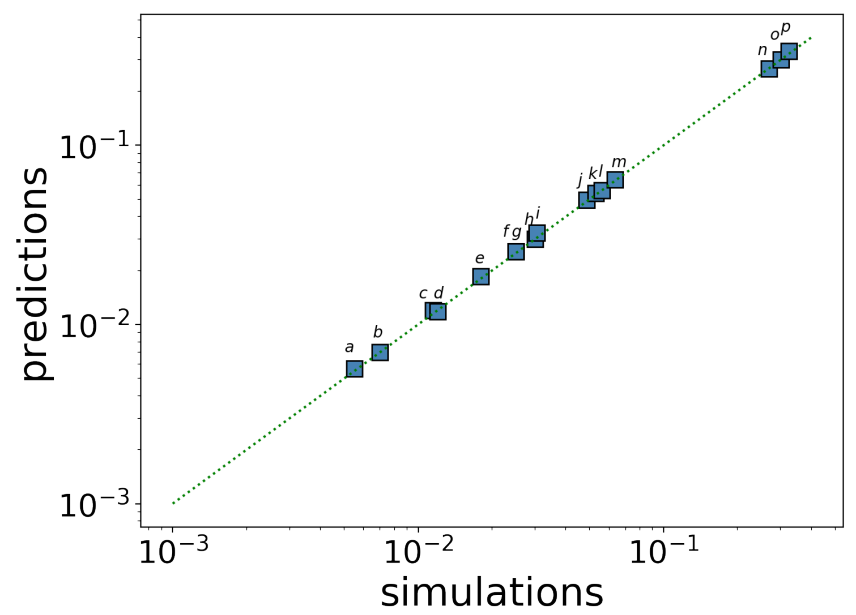

FIG. 7. Performance on more real-world networks. Identified versus simulated epidemic threshold with 16 real-world network data set. $a$ to $p$ represent Reactome, Facebook, AstroPh, Brightkite, Caida, Hamsterster-full, Hamster Friendships, Jazz Musicians, Pretty Good Privacy, Gnutella5, Gnutella6, Arenas-email, Gnutella4, US Power Grid, RV, Euroroad, respectively. Detail descriptions of the networks we use can be found in appendix. 
confusion scheme automatically detects if there's a phase transition in the input data and determines the epidemical threshold without any prior knowledge. Compared to our previous work, the proposed framework learns the topological and dynamical information simultaneously to give a better identification of the transition point. It is free from any kind of feature engineering, included the "sampling methods" we use in the previous work.

There still remains a lot of questions where further researches are needed. For instance, how to scale the input network up to a super large size while limiting the computational cost in a reasonable range; how to take a step forward to make the model fit different network topologies after a single training; how to apply our method in time-varying and multiplex networks, etc.

It is proved by plenty of experiments in 17 empirical data set that our machine learning framework performs great in almost every case. Our main contributions are two-fold. First, the proposition of our framework opened a new direction to learn and identify phase transition point of dynamics in complex networks, using machine learning techniques. Second, our work combines both topological and dynamical information of a network, exploring a new way to solve the threshold-related issues.

\section{ACKNOWLEDGMENTS}

This work was supported by the National Natural Science Foundation of China under Grant Nos. 11575041, 61802321, 11872182 and 11835003, the Natural Science Foundation of Shanghai under Grant No. 18ZR1412200, and the Science and Technology Commission of Shanghai Municipality under Grant No. 18dz2271000.

\section{APPENDIX}

The detailed information of the empirical networks:

CAIDA [53]. This is the undirected network of autonomous systems of the Internet from the CAIDA project, collected in 2007. Nodes are autonomous systems (AS) and edges represent communication.

Brightkite [54. This undirected network contains user-user friendship relations from Brightkite, a former location-based social network were users share their locations. A node represents a user and an edge indicates that a friendship exists between a pair of users.

Astro-Ph [53. This is the collaboration network of authors of scientific papers from the arXiv's Astrophysics (astro-ph) section. An edge between two authors represents a joint publication.
PGP (Pretty Good Privacy) [55. This is the interaction network of users of the Pretty Good Privacy (PGP) protocol. The network has only one giant connected component.

$R V$ (Route Views) [53. This is an undirected network of the autonomous system of the Internet.

Facebook [56]. This data set consists of "circles" (or "friend lists") from Facebook. Facebook data were collected from survey participants using this Facebook app. The data set includes nodal features (profiles), circles, and ego networks.

Gnutella [57. This is a sequence of snapshots of the Gnutella peer-to-peer file sharing network from August 2002. There are altogether nine snapshots of Gnutella network collected in August 2002. Nodes represent hosts in the Gnutella network and edges are connections between the hosts.

Reactome [58] This is a network of proteinprotein interactions in the species Homo sapiens, i.e., in Humans. The data is curated by the Reactome project, an open online database of biological pathways. Nodes represent protein and edges represent interactions.

Hamsterster-full [59] This Network contains friendships and familylinks between users of the website hamsterster.com. Nodes and edges represent users and userto-user relationship, respectively.

Hamsterster Friendships 60 This Network contains friendships and familylinks between users of the website hamsterster.com. Nodes and edges represent users and user-to-user relationship, respectively.

Jazz Musicians 61 This is the collaboration network between Jazz musicians. Each node is a Jazz musician and an edge denotes that two musicians have played together in a band. The data was collected in 2003. Nodes represent musicians and edges represent collaborations.

Arenas-email 62 This is the email communication network at the University Rovira i Virgili in Tarragona in the south of Catalonia in Spain. Nodes are users and each edge represents that at least one email was sent. The direction of emails or the number of emails are not stored.

US power grid [63] This undirected network contains information about the power grid of the Western States of the United States of America. An edge represents a power supply line. A node is either a generator, a transformator or a substation.

Euroroad 64] This is the international E-road network, a road network located mostly in Europe. The network is undirected; nodes represent cities and an edge between two nodes denotes that they are connected by an E-road.
[1] M. I. Jordan and T. M. Mitchell, Science 349, 255 (2015).

[2] L. Wang, Phys. Rev. B 94, 195105 (2016)
[3] T. Ohtsuki and T. Ohtsuki, J. Phys. Soc. Jpn. 85, 123706 (2016). 
[4] F. Schindler, N. Regnault, and T. Neupert, Phys. Rev. B 95, 245134 (2017)

[5] Y. Zhang, R. G. Melko, and E.-A. Kim, Phys. Rev. B 96, 245119 (2017).

[6] E. P. Van Nieuwenburg, Y.-H. Liu, and S. D. Huber, Nat. Phys. 13, 435 (2017).

[7] J. Carrasquilla and R. G. Melko, Nat. Phys. 13, 431 (2017).

[8] G. Carleo and M. Troyer, Science 355, 602 (2017).

[9] Y. Zhang and E.-A. Kim, Phys. Rev. Lett. 118, 216401 (2017)

[10] J. Liu, Y. Qi, Z. Y. Meng, and L. Fu, Phys. Rev. B 95, $041101(2017)$

[11] D.-L. Deng, X. Li, and S. Das Sarma, Phys. Rev. X 7, 021021 (2017)

[12] D.-L. Deng, X. Li, and S. Das Sarma, Phys. Rev. B 96, 195145 (2017)

[13] J. Venderley, V. Khemani, and E.-A. Kim, Phys. Rev. Lett. 120, 257204 (2018)

[14] A. C. Courville, I. Goodfellow, and Y. Bengio, Deep Learning (MIT Press, Cambridge MA, 2015).

[15] M. E. J. Newman, Networks: An Introduction (Oxford University Press, Oxford UK, 2010).

[16] S. N. Dorogovtsev, A. V. Goltsev, and J. F. F. Mendes, Rev. Mod. Phys. 80, 1275 (2008)

[17] B. Perozzi, R. Al-Rfou, and S. Skiena, in Proceedings of the 20th ACM SIGKDD International Conference on Knowledge Discovery and Data Mining (ACM, 2014) pp. 701-710.

[18] A. Grover and J. Leskovec, in Proceedings of the 22nd ACM SIGKDD International Conference on Knowledge Discovery and Data Mining (ACM, 2016) pp. 855-864.

[19] T. N. Kipf and M. Welling, arXiv preprint arXiv:1609.02907 (2016).

[20] D. Wang, P. Cui, and W. Zhu, in Proceedings of the 22nd ACM SIGKDD International Conference on Knowledge Discovery and Data Mining (ACM, 2016) pp. 1225-1234.

[21] W. L. Hamilton, R. Ying, and J. Leskovec, arXiv preprint arXiv:1709.05584 (2017).

[22] D. Liben-Nowell and J. Kleinberg, J. Ame. Soc. Info. Sci. Tech. 58, 1019 (2007).

[23] C. H. Ding, X. He, H. Zha, M. Gu, and H. D. Simon, in Data Mining, 2001. ICDM 2001, Proceedings IEEE International Conference on (IEEE, 2001) pp. 107-114.

[24] S. Bhagat, G. Cormode, and S. Muthukrishnan, in Social Network Data Analytics (Springer, 2011) pp. 115-148.

[25] R. Pastor-Satorras and A. Vespignani, Phys. Rev. Lett. 86, 3200 (2001).

[26] R. Pastor-Satorras and A. Vespignani, Phys. Rev. E 63, 066117 (2001).

[27] M. E. Newman, Phys. Rev. E 66, 016128 (2002).

[28] A. Vespignani, Science 325, 425 (2009).

[29] A. Vespignani, Nat. Phys. 8, 32 (2012).

[30] R. Pastor-Satorras, C. Castellano, P. Van Mieghem, and A. Vespignani, Rev. Mod. Phys. 87, 925 (2015).

[31] M. De Domenico, C. Granell, M. A. Porter, and A. Arenas, Nat. Phys. 12, 901 (2016).

[32] W. Wang, M. Tang, H. E. Stanley, and L. A. Braunstein, Rep. Prog. Phys. 80, 036603 (2017).

[33] S. C. Ferreira, C. Castellano, and R. Pastor-Satorras, Phys. Rev. E 86, 041125 (2012).

[34] P. Shu, W. Wang, M. Tang, and Y. Do, Chaos 25, 063104 (2015)

[35] M. Boguná, C. Castellano, and R. Pastor-Satorras, Phys.
Rev. Lett. 111, 068701 (2013).

[36] Q. Ni, M. Tang, Y. Liu, and Y.-C. Lai (unpublished, 2019).

[37] A. J.-P. Tixier, G. Nikolentzos, P. Meladianos, and M. Vazirgiannis, arXiv preprint arXiv:1708.02218 (2017).

[38] W. R. Tobler, Economic geography 46, 234 (1970).

[39] B. Perozzi, R. Al-Rfou, and S. Skiena, in Proceedings of the 20th ACM SIGKDD international conference on Knowledge discovery and data mining (ACM, 2014) pp. 701-710.

[40] J. Shlens, arXiv preprint arXiv:1404.1100 (2014).

[41] J. Zhou, G. Cui, Z. Zhang, C. Yang, Z. Liu, and M. Sun, arXiv preprint arXiv:1812.08434 (2018).

[42] Z. Wu, S. Pan, F. Chen, G. Long, C. Zhang, and P. S. Yu, arXiv preprint arXiv:1901.00596 (2019).

[43] M. M. Bronstein, J. Bruna, Y. LeCun, A. Szlam, and P. Vandergheynst, IEEE Signal Processing Magazine 34, 18 (2017).

[44] T. N. Kipf and M. Welling, arXiv preprint arXiv:1609.02907 (2016).

[45] N. Srivastava, G. Hinton, A. Krizhevsky, I. Sutskever, and R. Salakhutdinov, The journal of machine learning research 15, 1929 (2014).

[46] R. S. Sander, G. S. Costa, and S. C. Ferreira, Phys. Rev. E 94, 042308 (2016).

[47] D. P. Kingma and J. Ba, arXiv preprint arXiv:1412.6980 (2014).

[48] F. Bonahon, Low-dimensional geometry: From Euclidean surfaces to hyperbolic knots, Vol. 49 (American Mathematical Soc., 2009).

[49] A. Muscoloni, J. M. Thomas, S. Ciucci, G. Bianconi, and C. V. Cannistraci, Nature communications 8, 1615 (2017).

[50] F. Papadopoulos, M. Kitsak, M. Á. Serrano, M. Boguná, and D. Krioukov, Nature 489, 537 (2012).

[51] M. Nickel and D. Kiela, in Advances in neural information processing systems (2017) pp. 6338-6347.

[52] C. Castellano and R. Pastor-Satorras, Sci. Rep. 2, 371 (2012).

[53] J. Leskovec, J. Kleinberg, and C. Faloutsos, ACM Trans. Knowledge Dis. Data (TKDD) 1, 2 (2007).

[54] E. Cho, S. A. Myers, and J. Leskovec, in Proceedings of the 17th ACM SIGKDD International Conference on Knowledge Discovery and Data Mining (ACM, 2011) pp. 1082-1090.

[55] M. Boguñá, R. Pastor-Satorras, A. Díaz-Guilera, and A. Arenas, Phys. Rev. E 70, 056122 (2004).

[56] J. Leskovec and J. J. Mcauley, in Advances in Neural Information Processing Systems (2012) pp. 539-547.

[57] M. Ripeanu, I. Foster, and A. Iamnitchi, arXiv preprint cs/0209028 (2002).

[58] G. Joshi-Tope, M. Gillespie, I. Vastrik, P. D'Eustachio, E. Schmidt, B. de Bono, B. Jassal, G. Gopinath, G. Wu, L. Matthews, et al., Nucleic acids research 33, D428 (2005).

[59] KONECT, "Hamsterster full network dataset," (April, 2017).

[60] R. A. Rossi and N. K. Ahmed, in $A A A I$ (2015).

[61] P. M. Gleiser and L. Danon, Advances in complex systems 6, 565 (2003).

[62] R. Guimera, L. Danon, A. Diaz-Guilera, F. Giralt, and A. Arenas, Physical review E 68, 065103 (2003). 
[63] D. J. Watts and S. H. Strogatz, nature 393, 440 (1998).

[64] L. Šubelj and M. Bajec, The European Physical Journal B 81, 353 (2011). 